\title{
Intervenção Coronariana Percutânea em Artéria Coronariana Única em Paciente com Angina Instável de Alto Risco
}

\author{
Percutaneous Coronary Intervention in Single Coronary Artery in a Patient with Hig-Risk Unstable Angina \\ Hugo Alexandre Ross Yokoyama, Caio Mário de Almeida Pessoa, Fábio Cardoso de Carvalho, Ricardo Fernando de \\ Campos, Roberto Jorge da Silva Franco, Edson Antonio Bregagnollo \\ Hospital Estadual de Bauru e Faculdade de Medicina de Botucatu - UNESP - Bauru, SP - Botucatu, SP
}

No presente caso, relatamos a realização de angioplastia coronariana com implante de stent na artéria coronariana direita de paciente com quadro de angina instável de alto risco, portadora de artéria coronariana única com origem no seio coronariano direito. As artérias descendente anterior e circunflexa originavam-se isoladamente no terço proximal da artéria coronariana direita. Trata-se de rara anomalia coronariana com poucos relatos de intervenção coronariana percutânea na literatura. Este caso ilustra a necessidade da avaliação anatômica pormenorizada do trajeto das artérias coronarianas, precedendo a realização da angioplastia transluminal percutânea, objetivando-se a prevenção de complicações.
In the present case, we report the performance of coronary angioplasty with stent implantation in the right coronary artery of a patient with high-risk unstable angina and single coronary artery originating from the right coronary sinus. The anterior descending and circumflex arteries originated separately from the proximal third of the right coronary artery. This is a rare coronary anomaly and few reports of percutaneous coronary intervention are found in the literature. This case illustrates the need for a detailed anatomical assessment of the course of the coronary arteries prior to the performance of a percutaneous transluminal angioplasty, with the purpose of preventing complications.

\section{Introdução}

A artéria coronariana única (ACU) é descrita como uma artéria isolada, com origem na raiz da aorta, por meio de um óstio único e sem evidência de um segundo óstio, sendo então responsável pela irrigação de todo o coração, independentemente de sua distribuição ${ }^{1,2}$.

Anomalias das artérias coronárias podem causar problemas técnicos durante a angiografia e a angioplastia transluminal coronariana (ATC), sendo portanto necessário um conhecimento maior quanto ao procedimento e à completa avaliação da anatomia arterial coronariana para prevenção de complicações.

Relatamos o caso de A.T.C. com implante de stent em ramo coronariano direito distal, de paciente com ACU com quadro de angina instável após avaliação do trajeto proximal da artéria descendente anterior (DA).

\section{Relato do Caso}

Paciente de 69 anos, sexo feminino, branca, aposentada, tabagista, hipertensa e dislipidêmica, em uso domiciliar de AAS e propranolol, internada em razão de quadro de angina instável há dois dias. O eletrocardiograma (ECG) mostrava ritmo sinusal, freqüência cardíaca de 60 bpm e inversão da

\section{Palavras-chave \\ Angioplastia transluminal percutânea coronária, anomalias dos vasos coronários, angina instável.}

Correspondência: Hugo Alexandre Ross Yokoyama •

Av. Luiz Edmundo Corrijo Coube, 1-100 - Núcleo Geisel

17033-360 - Bauru, SP

E-mail: haross@cardiol.br

Artigo recebido em 05/04/06; revisado recebido em 12/06/06; aceito em 12/06/06. onda T na parede inferior. Durante os episódios dolorosos apresentava supradesnivelamento do segmento ST na parede inferior com melhora da repolarização após uso de nitrato sublingual (fig. 1). Os exames laboratoriais não mostraram alterações dos marcadores de lesão miocárdica.

A paciente foi submetida a coronariografia, que evidenciou a presença de $\mathrm{ACU}$ com origem no seio coronário direito. As artérias DA e circunflexa esquerda (CX) originavam-se isoladamente no terço proximal da artéria coronária direita (CD), a qual apresentava lesão obstrutiva significativa (95\%) em seu terço distal (fig. 2). Na ventriculografia esquerda observou-se discreto déficit contrátil por hipocinesia da parede inferior.

Após estudo do trajeto proximal da artéria DA com o uso de um catéter Pigtail posicionado em artéria pulmonar, optou-se por ATC com implante de stent coronário. O procedimento foi realizado por punção da artéria femoral utilizando-se cateter-guia JR 4 6F. Durante a seletivação da artéria coronariana única, a corda-guia 0,014 floppy progredia preferencialmente para a artéria DA. Após posicionamento adequado da corda-guia no leito distal da CD e pré-dilatação da lesão com balão 2,0 x $20 \mathrm{~mm}$, realizou-se implante de stent de 2,5 x $16 \mathrm{~mm}$ com sucesso primário e fluxo coronariano distal TIMI III (fig. 3, 4).

A paciente evoluiu sem intercorrências, recebendo alta após dois dias, com prescrição de ticlopidina (500 mg/dia por 30 dias), omeprazol (20 mg/dia), captopril (50 mg/dia), ácido acetilsalicílico (200 mg/dia) e metoprolol (50 mg/dia).

\section{Discussão}

Com a introdução e o desenvolvimento de novas técnicas de imagem do coração, a anatomia normal, as variações e 

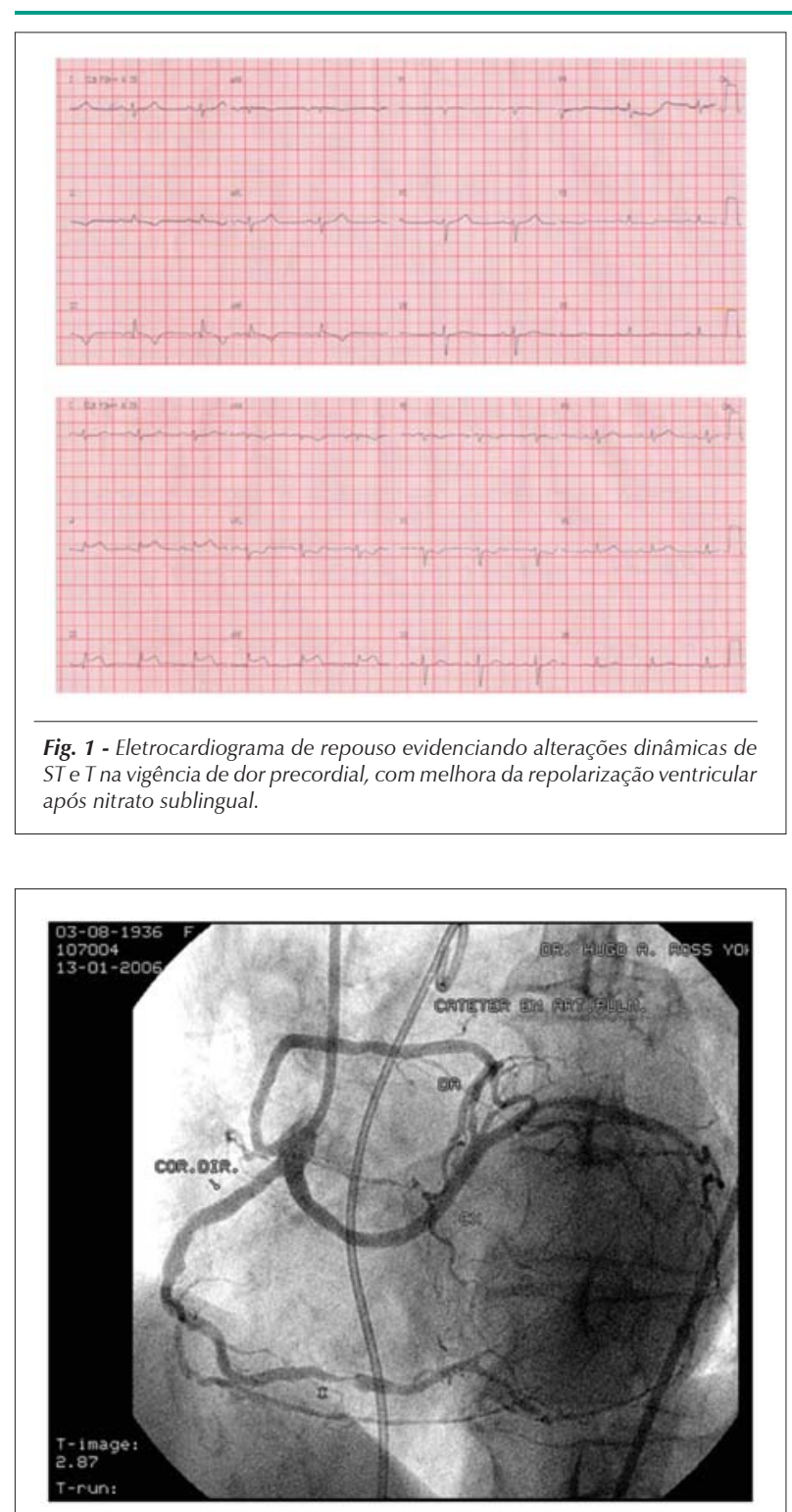

Fig. 2 - Angiografia na artéria coronariana única - projeção OAE.

anomalias das artérias coronarianas têm assumido um novo significado. A incidência de todas as anomalias coronarianas é de $0,23 \%$ em achados de autópsia, e varia de $0,3 \%$ a $12 \%$ em achados de angiografias ${ }^{3}$.

As anomalias coronarianas podem ser classificadas $\mathrm{Como}^{2}$ : 1) as que alteram a perfusão miocárdica (fístulas, origem da coronária esquerda a partir da artéria pulmonar, estenose ou atresia congênita da coronária, origem a partir do seio contralateral com passagem entre a aorta e o trato ventricular direito); 2) as que não comprometem a perfusão miocárdica (origem da artéria circunflexa a partir do seio aórtico direito, origem da descendente anterior esquerda a partir do seio aórtico direito, artéria coronariana única, origem das três artérias coronarianas a partir dos seios direito ou esquerdo através de óstios separados e origem alta das artérias coronárias). Em particular, a origem e o curso anômalos da

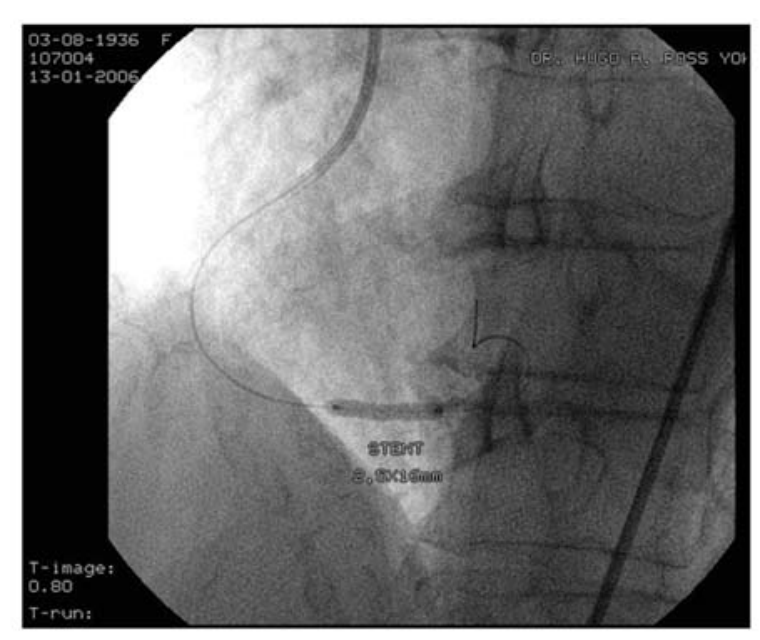

Fig. 3 - Liberação do stent 2,5 x 16 mm sobre a lesão-alvo.

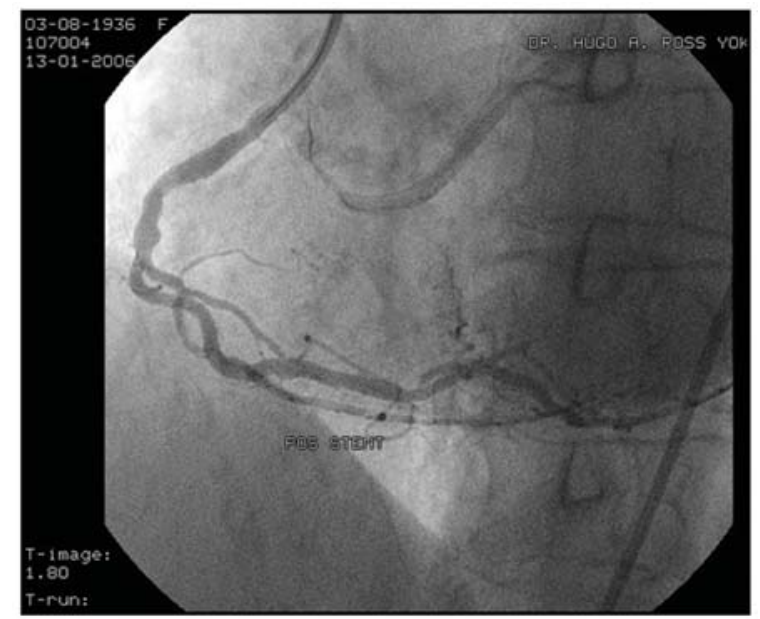

Fig. 4 - Resultado final.

artéria coronária esquerda, classicamente considerados como malformação menor, têm sido implicados como causadores de isquemia miocárdica e morte súbita ${ }^{1,2}$. A incidência de ACU é de $0,024 \%$ quando isolada, e de $0,03 \%$ a $0,04 \%$ quando associada a outras anormalidades cardíacas.

O prognóstico nos casos de ACU, como anomalia isolada, varia de excelente a reservado, conforme sua distribuição anatômica, incluindo risco de morte súbita; $15 \%$ dos pacientes apresentam problemas cardíacos graves antes dos 40 anos. Esse risco é justificado pelo trajeto da coronária anômala através da base do coração até atingir seu território de distribuição, o que pode levar à compressão por outras estruturas ou à angulação/acotovelamento de sua origem ${ }^{1,2}$. Pacientes com a artéria coronariana esquerda originando-se do seio coronário direito apresentam taxa de mortalidade elevada antes dos 20 anos (59\%). As mortes geralmente ocorrem durante ou após a prática de atividade física intensa ${ }^{1}$. Assim, a avaliação do trajeto do vaso aberrante é de fundamental importância 


\section{Relato de Caso}

para a determinação do prognóstico, uma vez que o trajeto entre a via de saída do ventrículo direito e a aorta apresenta prognóstico reservado se o tratamento cirúrgico não for instituído. A forma adequada para essa avaliação consiste em posicionar um catéter na artéria pulmonar e depois realizar a angiografia coronariana para a determinação se o trajeto da coronária anômala é anterior ou posterior à artéria pulmonar. Nos casos com trajeto anterior, o prognóstico é benigno.

Nos pacientes com ACU, os mecanismos de morte súbita não são totalmente conhecidos, visto que a maioria dos dados provém de achados de autópsias. Os principais mecanismos de morte súbita apontados na literatura incluem: 1) compressão do trajeto anômalo entre a aorta e a artéria pulmonar; 2) a ACU sairia da aorta em posição oblíqua, possibilitando um acotovelamento próximo ao óstio que durante a expansão da aorta poderia obstruir o óstio coronário; 3) presença de angulação incomum da ACU e curso proximal tortuoso, determinando padrão de fluxo turbulento e aterosclerose acelerada ${ }^{1-4}$.

Em uma revisão de 178 casos de anomalias coronarianas submetidas a tratamento cirúrgico, Reul e cols. ${ }^{5}$ relatam que 15 pacientes apresentavam anomalias de origem da coronária direita ou esquerda proveniente do seio coronário contralateral. As técnicas cirúrgicas utilizadas foram o reimplante da artéria coronariana anômala, a ligadura da coronária seguida de enxerto com safena e anastomose com artéria mamária interna. Questionamentos quanto à melhor técnica geralmente são feitos, visto que a maioria desses pacientes é jovem e os enxertos com safenas apresentam degeneração maior que as anastomoses de mamária.

Neste relato, a anomalia coronariana observada está de acordo com a classificação de Shirani e Roberts ${ }^{6}$. Seguindo essa classificação, este seria um caso IID1, no qual temos um óstio solitário no seio coronário direito, a artéria CX com origem na porção proximal da $C D$ seguindo para a esquerda por trás da aorta e a artéria DA também surgindo da CD proximal e seguindo para a esquerda anteriormente à artéria pulmonar ${ }^{7}$.

Topaz e cols. ${ }^{8}$ relatam o tratamento de cinco casos de lesões ateroscleróticas coronarianas com a angioplastia em pacientes com anomalias coronárias. Nestes casos, a avaliação

\section{Referências}

1. Angelini P, Velasco JA, Flamm S. Coronary anomalies: incidence, pathophysiology, and clinical relevance. Circulation. 2002;105: 2249-54.

2. Popma JJ. Coronary angiography and intravascular imaging. In: Zipes DP, Libby P, Bonow RO, Braunwald E, eds. Heart disease: a textbook of cardiovascular medicine. 7th ed. Philadelphia: Elsevier Saunders; 2005. p. 423-55.

3. Vilallonga JR. Anatomical variations in the coronary arteries. II. Less prevalent variations: Coronary anomalies. Eur J Anat. 2004; 8: 39-53.

4. Lim MJ, Forsberg MJ, Lee R, Kern MJ. Hemodynamic Abnormalities across an anomalous left main coronary artery assessment: Evidence for a dynamic ostial obstruction. Cath Cardiovasc Interv. 2004; 63: 294-8.

5. Reul RM, Cooley DA, Hallman GL. Surgical treatment of coronary artery anomalies (Report of a 37 1/2 -year experience at the Texas Heart Institute). Tex Heart Inst J. 2002; 29: 299-307.

6. Shirani J, Roberts WC. Solitary coronary ostium in aorta in the absence of cuidadosa da configuração do orifício coronário, angulação proximal, trajeto do vaso e localização da lesão estenótica foram primordiais para o sucesso do procedimento, sendo a seleção adequada do cateter-guia e o avanço do balão na porção proximal do vaso anômalo os maiores determinantes de sucesso. Em nosso caso, após cuidadosa avaliação do trajeto da coronária anômala, optou-se pela intervenção utilizando-se cateter-guia tipo JR. No início do procedimento houve certa dificuldade, pois o cateter escolhido apresentava a tendência de desviar-se da origem da artéria DA anômala na porção proximal da artéria CD e, com isso, a corda-guia 0,014 não atingia o ramo coronariano direito. O direcionamento da ponta do cateter para a artéria CX possibilitou a manipulação da corda-guia 0,014 até o leito distal da artéria CD. A partir de então, o procedimento não apresentou dificuldades técnicas.

Deve-se salientar que em casos de obstruções ateroscleróticas associadas a anomalias coronarianas, o estudo adequado do trajeto do vaso anômalo é fundamental, visto que, caso diagnosticado trajeto entre a artéria pulmonar e a aorta, o tratamento cirúrgico é a opção preferencial para os casos eletivos.

Praharaj e Ray ${ }^{9}$ relataram dois casos de angioplastia coronariana com implante de stent em artéria coronariana direita anômala originando-se do seio coronariano esquerdo. Nesse relato, salientam a necessidade da avaliação completa da anatomia coronariana precedendo a realização da intervenção, para a prevenção de complicações.

Nas diretrizes para ATC da Sociedade Européia da Cardiologia, no item que avalia a ATC em situações especiais não são apresentadas orientações quanto a condutas que deveriam ser utilizadas em pacientes com anomalias coronarianas ${ }^{10}$. No entanto, a normatização definitiva é difícil, dadas a raridade e as variações anatômicas dessas anomalias. No momento, acreditamos que cada caso deva ser analisado e tratado individualmente, de acordo com as variações anatômicas observadas.

\section{Potencial Conflito de Interesses}

Declaro não haver conflitos de interesses pertinentes. major congenital cardiovascular anomalies. J Am Coll Cardiol. 1993; 21: 137-43.

7. Neil DAH, Bonser RS, Townend JN. Coronary arteries from a single coronary ostium in the right coronary sinus: a previously unreported anatomy. Heart. 2000; 83: e9.

8. Topaz O, DiSciascio G, Goudreau E, Cowley MJ, Nath A, Kohli RS, et al. Coronary angioplasty of anomalous coronary arteries:notes on technical aspects. Cathet Cardiovasc Diagn. 1990; 21: 106-11.

9. Praharaj TK, Ray G. Percutaneous transluminal coronary angioplasty with stenting of anomalous right coronary artery originating from left sinus of valsalva using the voda guiding catheter: a report of two cases. Indian Heart J. 2001; 53: 79-82.

10. Silber S, Albertsson P, Aviles FF, Camici PG, Colombo A, Hamm C, et al. Guidelines for percutaneous coronary interventions. The Task Force for Percutaneous Interventions of the European Society of Cardiology. Eur Heart J. 2005 ; 26 : 804-47. 